graphic image from which the FFT was calculated. FFT images correspond to the module of two-dimensional (2D)-FFT (displayed in linear scale), calculated by using the WSxM software developed by R. Fernández et al. [19].

Received: November 5, 2002 Final version: February 13, 2003

[1] S. K. Moore, IEEE Spectrum 2002, 39, 55.

[2] R. D. McCullough, R. D. Lowe, J. Chem. Soc., Chem. Commun. 1992, 1 , 70 .

[3] T. A. Chen, R. D. Rieke, J. Am. Chem. Soc. 1992, 114, 10087.

[4] H. E. Katz, Z. Bao, S. L. Gilat, Acc. Chem. Res. 2001, 34, 359.

[5] Z. Bao, A. Dodabalapur, A. J. Lovinger, Appl. Phys. Lett. 1996, 69, 4108.

[6] H. Sirringhaus, N. Tessler, R. H. Friend, Science 1998, 280, 1741.

[7] H. Sirringhaus, P. J. Brown, R. H. Friend, M. M. Nielsen, K. Bechgaard, B. M. W. Langeveld-Voss, A. J. H. Spiering, R. A. J. Janssen, E. W. Meijer, P. Herwig, D. M. de Leeuw, Nature 1999, 401, 685.

[8] K. E. Aasmundtveit, E. J. Samuelsen, M. Guldstein, C. Steinsland, O. Flornes, C. Fagermo, T. M. Seeberg, L. A. A. Pettersson, O. Inganäs, R. Feidenhans, S. Ferrer, Macromolecules 2000, 33, 3120.

[9] H. Sirringhaus, P. J. Brown, R. H. Friend, M. M. Nielsen, K. Bechgaard, B. M. W. Langeveld-Voss, A. J. H. Spiering, R. A. J. Janssen, E. W. Meijer, Synth. Met. 2000, 111-112, 129.

[10] T. J. Prosa, J. Moulton, A. J. Heeger, M. J. Winokur, Macromolecules 1999, 32, 4000.

[11] S. S. Sheiko, M. Möller, Chem. Rev. 2001, 101, 4099.

[12] P. Samorí, J. P. Rabe, J.Phys.: Condens. Matter. 2002, 14, 9955

[13] E. Mena-Osteritz, A. Meyer, B. M. W. Langeveld-Voss, R. A. J. Janssen, E. W. Meijer, P. Bäuerle, Angew. Chem. Int. Ed. 2000, 39, 2680.

[14] E. Mena-Osteritz, Adv. Mater. 2002, 14, 609.

[15] Y. Okawa, M. Aono, Nature 2001, 409, 683.

[16] Y. Okawa, M. Aono, J. Chem. Phys. 2001, 115, 2317

[17] H. Kasai, H. Tanaka, S. Okada, H. Oikawa, T. Kawai, H. Nakanishi, Chem. Lett. 2002, 697.

[18] J. Liu, R. S. Loewe, R. D. McCullough, Macromolecules 1999, 32, 5777

[19] R. Fernández, I. Horcas, P. Colilla, M. Rodriguez, M. Godinez, K. Hu, M. Pereira, S. Preito, L. F. Robledano, R. Pérez, WSxM version 3.0, Nanotec Electronica, Madrid, Spain; www.nanotec.es

\section{Highly Efficient Red Electrophosphorescent Devices Based on Iridium Isoquinoline Complexes: Remarkable External Quantum Efficiency Over a Wide Range of Current**}

\author{
By Ying-Ju Su, Heh-Lung Huang, Chien-Le Li, \\ Chin-Hsiung Chien, Yu-Tai Tao,* Pi-Tai Chou, \\ Swarup Datta, and Rai-Shung Liu*
}

Organic light-emitting devices (OLEDs) hold great potential for application in flat-panel displays due to rapid progress in material design and device fabrication in recent years. ${ }^{[1-4]}$ Red, green, and blue emissions of matching efficiencies are

[*] Prof. R.-S. Liu, Y.-J. Su, H.-L. Huang, C.-L. Li, S. Datta Department of Chemistry, National Tsinghua University Hsinchu, 30043, Taiwan (ROC)

E-mail: rsliu@mx.nthu.edu.tw

Prof. Y.-T. Tao, C.-H. Chien

Department of Chemistry, Academic Sinica

Nankan, 10115, Taiwan (ROC)

E-mail: ytt@gate.sinica.edu.tw

Prof. P.-T. Chou

Department of Chemistry, National Taiwan University

Taipei, 10617, Taiwan (ROC)

[**] This work is supported by the National Science Council (NSC 91-2622M007-001) and Ministry of Education, Taiwan, ROC. required for a full-color display. Greater success has been achieved in the development of a green emitter than that of red one in both fluorescent and phosphorescent displays. For example, devices based on green fluorescent dopant $\mathrm{C}_{545 \mathrm{~T}^{[5]}}$ can reach a luminance efficiency $>10 \mathrm{~cd} \mathrm{~A}^{-1}$. In contrast, the best red OLEDs reported ${ }^{[6-10]}$ can only reach $\sim 3 \mathrm{~cd} \mathrm{~A}^{-1}$ and external quantum efficiencies less than $1 \%$. OLEDs based on phosphorescent materials ${ }^{[11]}$ can significantly improve electroluminescence performance because both singlet and triplet excitons can be harvested for light emission. Theoretically, the internal quantum efficiency of phosphorescent emitters can approach $100 \%$. Unfortunately, most of phosphorescent emitters have a long lifetime ( $>10 \mu \mathrm{s})$, which results in saturation of emissive sites at increasing currents. The first red phosphorescent material was reported for complexes of the type $\mathrm{EuL}_{3}$, where L denotes a ligand. ${ }^{[12]}$ The long lifetime of the electronically excited europium(III) $(350 \mu \mathrm{s})$ leads to dominant triplet-triplet (T-T) annihilation at high currents, and the maximum efficiency is only $1.4 \%$. Although platinum(II) octaethylporphyrin (PtOEP) doped into 4,4,N, $N^{\prime}$-dicarbazolebiphenyl (CBP) shows an external quantum efficiency $\eta_{\text {ext }}=5.6 \%$, for a red phosphor, ${ }^{[4,13]}$ the occurrence of $\mathrm{T}-\mathrm{T}$ annihilation is inevitable at high currents $\left(\eta_{\text {ext }}=0.5 \%\right.$ at $\left.100 \mathrm{~mA} \mathrm{~cm}^{-2}\right)$ due to its relatively long phosphorescent lifetime $(50-80 \mu \mathrm{s})$. Recently, a more efficient red phosphor has been reported, $\operatorname{Ir}(\mathrm{btp})_{2}(\mathrm{acac})^{[14]}\left(\mathrm{btp}=2-\left(2^{\prime}\right.\right.$-benzo[4.5-a]thienyl)pyridinato; acac $=$ acetylacetonate $)$, that achieved an efficiency of $\eta_{\text {ext }}=7.0 \%$ at low current. The relatively short phosphorescent lifetime ( $4 \mu \mathrm{s})$ significantly improves the external efficiency at high currents $\left(\eta_{\text {ext }}=2.5 \%\right.$ at $\left.100 \mathrm{~mA} \mathrm{~cm}^{-2}\right)$. A short phosphorescence lifetime is crucial for the performance of a phosphorescent material, particularly its maximum brightness and efficiency at high currents. In this communication, we wish to report a series of efficient red phosphorescent emitters based on iridium 1-(phenyl)isoquinoline complexes which show strong electroluminescence (EL) brightness and efficiency, even at high currents.

Scheme 1 outlines the synthetic protocol for red phosphorescent complexes: $\operatorname{Ir}(\mathrm{piq})_{2}(\mathrm{acac}), \operatorname{Ir}(\text { piq-F })_{2}$ (acac), and $\operatorname{Ir}(\text { piq-F })_{3}$, with piq and piq-F representing the 1-(phenyl)isoquinoline and 2-(4'-fluorophenyl)isoquinoline ligands, respectively. The para-fluoro substituent of $\operatorname{Ir}(\text { piq-F })_{2}$ (acac) and $\operatorname{Ir}(\text { piq-F })_{3}$ is used to tune emission wavelength without loss of performance efficiency. ${ }^{[15]}$ 1-(Phenyl)isoquinoline ligand was prepared from the reaction of isoquinoline with the corresponding phenyl magnesium bromide. The complexes Ir$(\text { piq })_{2}$ (acac) and $\operatorname{Ir}(\text { piq-F })_{2}$ (acac) were prepared via treatment of diiridium precursors ${ }^{[16]}$ with acetylacetone in the presence of $\mathrm{Na}_{2} \mathrm{CO}_{3}$. The tris-chelated complex $\operatorname{Ir}(\text { piq-F })_{3}$ was obtained from heating the diiridium complexes with $\mathrm{AgCF}_{3} \mathrm{SO}_{3}$ in the presence of a five-fold amount of piq-F at $200{ }^{\circ} \mathrm{C}$ for $24 \mathrm{~h}$. We also prepared complex with an iridium center cyclometalated with a 5-(4'-fluorophenyl)-(3,4-benzoquinoline) ligand, $\operatorname{Ir}(\mathrm{pbq}-\mathrm{F})_{2}$ (acac), which is expected to show red-shifted EL. ${ }^{1} \mathrm{H}$ and ${ }^{13} \mathrm{C}$ NMR, IR, mass, and elemental analyses are consistent with the proposed structures. 
(1)<smiles>[X]c1ccc([N+](=O)C(C)(Br)Br)cc1</smiles><smiles>[X]c1ccc(-c2nccc3ccccc23)cc1</smiles>

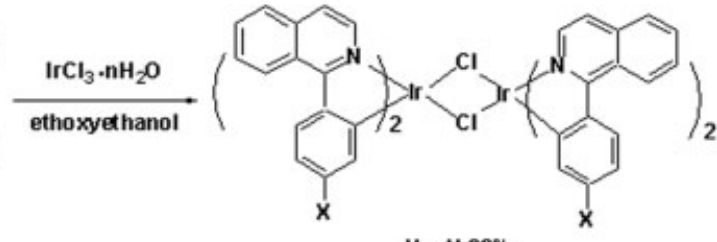

$X=H(p i q), 41 \%$

$\mathrm{F}$ (piq-F), $50 \%$

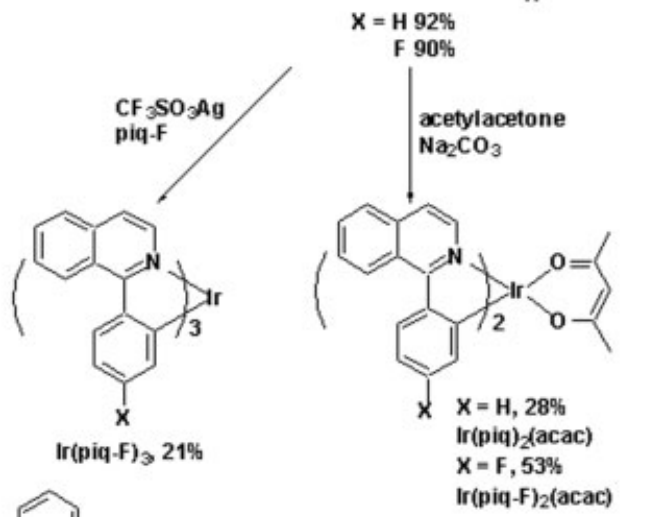

(2)<smiles>Fc1ccc(-c2nc3ccccc3c3ccccc23)cc1</smiles>

\section{(1) $\mathrm{IrCl}_{3} \mathrm{nH}_{2} \mathrm{O}$ ethoxyethanol \\ (2) acetylacetone $\mathrm{Na}_{2} \mathrm{CO}_{3}$}

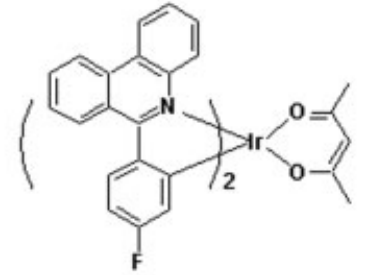

$\operatorname{Ir}(\mathrm{pbq}-\mathrm{F})_{2}(\mathrm{acac})$

Scheme 1. Synthesis of the complexes.

Table 1 and Figure 1 show the photophysical properties of these four iridium species. By comparison to the free 2-phenyisoquinoline derivatives, the bands below $360 \mathrm{~nm}$ were assigned to the spin-allowed ${ }^{1} \pi-\pi^{*}$ transition of cyclometalated ligand, and the band around $400 \mathrm{~nm}$ can be assigned to spinallowed metal-ligand charge transfer band ( ${ }^{1}$ MLCT) with $\varepsilon=1200-8210 \mathrm{M}^{-1} \mathrm{~cm}^{-1}$. The most surprising feature for these complexes is the strong intensity of the band around $450 \mathrm{~nm}$, which can be assigned to the spin-forbidden ${ }^{3} \mathrm{MLCT}$ band. ${ }^{[17,18]}$ For these complexes, the extinction coefficients of this ${ }^{3}$ MLCT band lie in the range $\varepsilon=3043-9210 \mathrm{M}^{-1} \mathrm{~cm}^{-1}$, and this information indicates an efficient spin-orbit coupling that is prerequisite for phosphorescent emission. The PL spectrum of $\operatorname{Ir}(\text { piq })_{2}$ (acac) in $\mathrm{CH}_{2} \mathrm{Cl}_{2}$ shows an emission band at $622 \mathrm{~nm}$, whereas $\operatorname{Ir}(\text { piq-F })_{2}($ acac $)$ and $\operatorname{Ir}(\text { piq-F })_{3}$ are blue-

Table 1. Photophysical data of iridium complexes.

\begin{tabular}{|c|c|c|c|c|}
\hline Complex & $\begin{array}{c}\text { Absorption [a] } \\
{[\mathrm{nm}]}\end{array}$ & $\begin{array}{c}\text { Emission } \\
\text { qutanum yield[a] }\end{array}$ & $\tau_{\text {obs }}$ & $\tau_{\mathrm{r}}$ \\
\hline $\operatorname{Ir}(\text { piq })_{2}(\mathrm{acac})$ & $\begin{array}{c}226,286,336, \\
350,377,477\end{array}$ & 0.2 & $1.67[\mathrm{a}](0.44[\mathrm{~b}]) \mu \mathrm{s}$ & 8.35 \\
\hline $\operatorname{Ir}(\text { piq-F })_{2}(\mathrm{acac})$ & $\begin{array}{c}229,289,335, \\
352,398,452\end{array}$ & 0.33 & $1.20(0.30) \mu \mathrm{s}$ & 3.64 \\
\hline $\operatorname{Ir}(\text { piq-F })_{3}$ & $\begin{array}{c}228,283,312, \\
343,396,456\end{array}$ & 0.15 & $2.46(0.15) \mu \mathrm{s}$ & 16.4 \\
\hline $\operatorname{Ir}(\mathrm{pbq}-\mathrm{F})_{2}(\mathrm{acac})$ & $\begin{array}{c}252,350,378, \\
453\end{array}$ & 0.16 & $1.96(0.56) \mu \mathrm{s}$ & 12.3 \\
\hline
\end{tabular}

[a] Data obtained in $\mathrm{CH}_{2} \mathrm{Cl}_{2}$. [b] Lifetime measured in solid film.

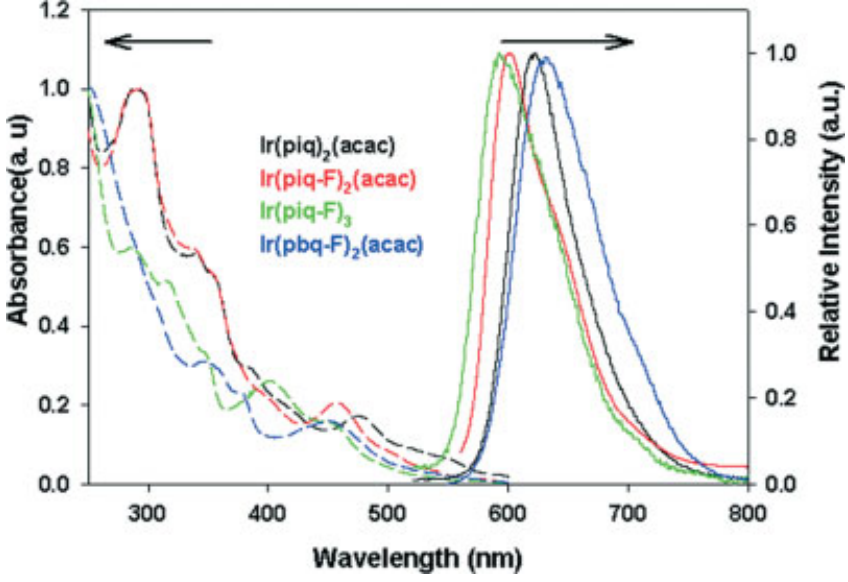

Fig. 1. UV and PL spectra of Ir complexes in $\mathrm{CH}_{2} \mathrm{Cl}_{2}$.

shifted to 600 and $595 \mathrm{~nm}$ respectively. The complex $\operatorname{Ir}(\mathrm{pbq}-$ $\mathrm{F})_{2}$ (acac) exhibits the largest bathochromic emission with a maximum at $631 \mathrm{~nm} \cdot{ }^{[14]}$ For these red phosphors, the observed lifetimes are ca. 1.2-2.5 $\mu$ s in $\mathrm{CH}_{2} \mathrm{Cl}_{2}$, considerably shorter than that of $\operatorname{Ir}(\mathrm{btp})_{2}$ (acac) $(6 \mu$ s in tetrahydrofuran (THF)). In the solid state, the lifetime is even shorter $(0.15-0.56 \mu \mathrm{s})$, possibly due to the quenching associated with molecular packing. The emission quantum efficiencies of the complexes in $\mathrm{CH}_{2} \mathrm{Cl}_{2}$ lie within the range $0.15-0.33$, with $\operatorname{Ir}(\text { piq-F })_{2}$ (acac) being the highest. Accordingly, the radiative lifetimes $\left(\tau_{\mathrm{r}}\right)$ are 
deduced to be as short as 3.6-16.4 $\mu$ s (see Table 1), which correlates well with the unusually large extinction coefficients measured for the ${ }^{3}$ MLCT bands.

OLEDs using iridium complexes were fabricated by highvacuum $\left(10^{-6}\right.$ torr) thermal evaporation onto pre-cleaned glass substrates. Figure 2 shows the configuration of the devices, in which CBP acted as a host material for the com-

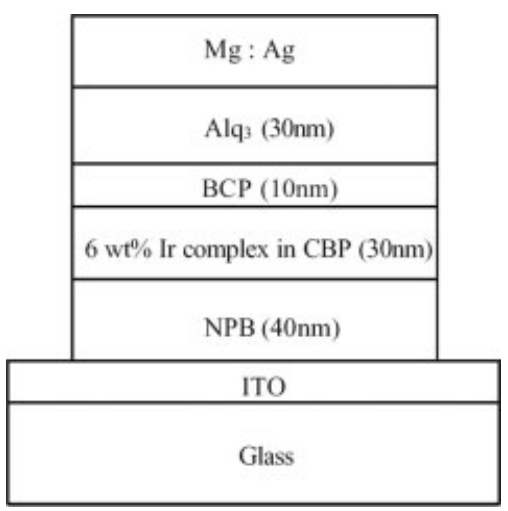

Fig. 2. Configuration of the devices used, including typical layer thicknesses.

plexes, bathocuproine (BCP) as a hole blocker, and 4,4' bis[ $N$-(naphthyl)- $N$-phenyl-amino]biphenyl (NPB) and tris(8-hydroxyquinoline)aluminum(III) $\left(\mathrm{Alq}_{3}\right)$ as a hole-transport layer and an electron-transport layer, respectively.

All four devices exhibited bright orange-red to orange emissions, which were independent of the applied voltages from $6 \mathrm{~V}$ to $12 \mathrm{~V}$. Figure 3 shows the EL spectra of devices based on these four iridium species at a current density of $J=20 \mathrm{~mA} \mathrm{~cm}^{-2}$. The corresponding CIE (Commission Internationale de l'Eclairage) coordinates are $x=0.68, y=0.32$ for $\operatorname{Ir}(\text { piq })_{2}($ acac $), x=0.61, y=0.36$ for $\operatorname{Ir}(\text { piq-F })_{2}($ acac $), x=0.61$, $y=0.38$ for $\operatorname{Ir}(\text { piq-F })_{3}$, and $x=0.65, y=0.33$ for $\operatorname{Ir}(\mathrm{pbq})_{2}(\mathrm{acac})$, respectively (see Table 2 ).

Using the above devices, EL data for the complexes were acquired, as summarized in Table 2. Complexes $\operatorname{Ir}(\text { piq })_{2^{-}}$ (acac), $\operatorname{Ir}(\text { piq-F })_{2}\left(\right.$ acac), and $\operatorname{Ir}(\text { piq-F })_{3}$ show better performance than $\operatorname{Ir}(\mathrm{pbq})_{2}(\mathrm{acac})$ in both maximum brightness and quantum efficiency. Using $\operatorname{Ir}(\text { piq-F })_{2}$ (acac), a maximum brightness of $27130 \mathrm{~cd} \mathrm{~m}^{-2}$ was achieved at a current density of $J=400 \mathrm{~mA} \mathrm{~cm}^{-2}$. The external quantum efficiency $\eta_{\text {ext }}$ of $\operatorname{Ir}($ piqF) $)_{2}$ (acac) was $8.67 \%$ at $J=20 \mathrm{~mA} \mathrm{~cm}^{-2}$, where a maximum of luminance efficiency (LE) of $4.73 \mathrm{~lm} \mathrm{~W}^{-1}$ and a power efficiency (PE) of $13.7 \mathrm{~cd} \mathrm{~A}^{-1}$ were obtained (see Fig. 4). The external quantum efficiency decreased very slowly with increasing current At $J=100 \mathrm{~mA} \mathrm{~cm}^{-2}$, the value of $\eta_{\text {ext }}$ remained as high as $6.84 \%$, with a luminance and power efficiency $2.83 \mathrm{~lm} \mathrm{~W}^{-1}$ and $10.8 \mathrm{~cd} \mathrm{~A}^{-1}$ respectively. At high current $J=400 \mathrm{~mA} \mathrm{~cm}^{-2}, \eta_{\text {ext }}$ was still as high as

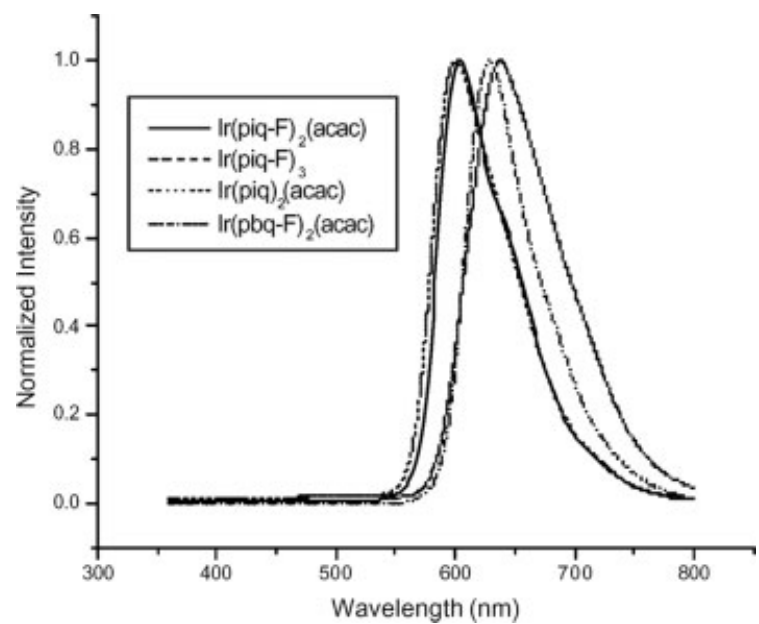

Fig. 3. EL spectra of four iridium species at $J=20 \mathrm{~mA} \mathrm{~cm}^{-2}$.

$4.20 \%$, with $\mathrm{LE}=1.42 \mathrm{~lm} \mathrm{~W}^{-1}$ and $\mathrm{PE}=6.75 \mathrm{~cd} \mathrm{~A}^{-1}$. In comparison, the maximum brightness of a known complex btp ${ }_{2} \mathrm{Ir}-$ (acac) $^{[14]}$ was reported to be only ca. $6800 \mathrm{~cd} \mathrm{~m}^{-2}$ at $J=690 \mathrm{~mA} \mathrm{~cm}^{-2}$ in spite of its external quantum efficiency of $\eta_{\text {ext }}=7.0$ at $J=0.01 \mathrm{~mA} \mathrm{~cm}^{-2}$. The $\eta_{\text {ext }}$ value for $b_{2} \operatorname{Ir}(\mathrm{acac})$ also drops more quickly with its increasing current, $\eta_{\mathrm{ext}}=4.4$ and 1.3 at $J=20$ and $400 \mathrm{~mA} \mathrm{~cm}^{-2}$ respectively. The data in $\mathrm{Ta}-$ ble 2 reveal that $\operatorname{Ir}(\mathrm{piq})_{2}(\mathrm{acac})$ and $\operatorname{Ir}(\text { piq-F })_{3}$ are equally efficient as $\operatorname{Ir}(\text { piq-F })_{2}(\mathrm{acac})$ in terms of maximum brightness, power, luminance, and external quantum efficiency. The role of the para-fluoro substituent of $\operatorname{Ir}(\text { piq-F })_{2}(\mathrm{acac})$ and $\operatorname{Ir}($ piq$\mathrm{F})_{3}$ is to tune the luminance towards high frequency without loss of EL efficiency. One notable feature for $\operatorname{Ir}(\text { piq })_{2}(\mathrm{acac})$ is the exceptionally high external quantum efficiency $\eta_{\mathrm{ext}}=7.00$ at $J=400 \mathrm{~mA} \mathrm{~cm}^{-2}$, corresponding to a loss of only $28 \%$ from its maximum value $\eta_{\mathrm{ext}}=9.71$ at $J=38 \mathrm{~mA} \mathrm{~cm}^{-2}$.

In summary, we have reported a series of high-efficiency and high-brightness red phosphors based on iridium complexes bearing a 1-(phenyl)isoquinoline framework. ${ }^{[19]}$ All studied complexes exhibit prominent EL emission in the range 600$630 \mathrm{~nm}$. Unlike normal iridium phosphor complexes, remarkably high efficiency can be maintained at high currents with a

Table 2. Electrophosphorescence data for iridium complexes.

\begin{tabular}{ccccccc}
\hline Parameter & $\begin{array}{c}\text { Brightness } \\
{\left[\mathrm{cd} \mathrm{m}^{-2}\right]}\end{array}$ & $\begin{array}{c}\text { EQE } \\
{[\%]}\end{array}$ & $\begin{array}{c}\text { PE } \\
{\left[\mathrm{cd} \mathrm{A}^{-1}\right]}\end{array}$ & $\begin{array}{c}\text { LE } \\
{\left[\mathrm{lm} \mathrm{W}^{-1}\right]}\end{array}$ & $\begin{array}{c}\text { Voltage } \\
{[\mathrm{V}]}\end{array}$ & $\begin{array}{c}\text { CIE } \\
\text { corordinates }\end{array}$ \\
\hline Ir(piq)2(acac) & $1514[\mathrm{a}]$ & 8.46 & 7.56 & 2.78 & 8.53 & $x=0.68$ \\
& $8224[\mathrm{~b}]$ & 9.21 & 8.22 & 2.34 & 11.01 & $y=0.32$ \\
& $24978[\mathrm{c}]$ & 7.00 & 6.08 & 1.88 & 13.92 & \\
Ir(piq- & 2744 & 8.67 & 13.72 & 4.73 & 9.11 & $x=0.61$ \\
F)2(acac) & 10808 & 6.84 & 10.82 & 2.82 & 12.01 & $y=0.36$ \\
& 26947 & 4.27 & 6.75 & 1.42 & 14.91 & \\
$\operatorname{Ir}($ piq-F)3 & 2078 & 5.81 & 10.36 & 3.80 & 8.56 & $x=0.61$ \\
& 9625 & 5.41 & 9.66 & 2.80 & 10.83 & $y=0.38$ \\
Ir(pbq & 25970 & 3.65 & 6.50 & 1.51 & 13.60 & \\
-F)2(acac) & 841 & 5.63 & 4.20 & 1.52 & 8.66 & $x=0.65$ \\
& 3788 & 5.10 & 3.80 & 1.08 & 11.12 & $y=0.33$ \\
& 10734 & 3.66 & 2.65 & 0.60 & 13.93 &
\end{tabular}

For each parameter, the data in different rows correspond to those measured at different current densities: [a] $J=20 \mathrm{~mA} \mathrm{~cm}^{-2}$. [b] $J=100 \mathrm{~mA} \mathrm{~cm}^{-2}$. [c] $J=400 \mathrm{~mA} \mathrm{~cm}^{-2}$. 


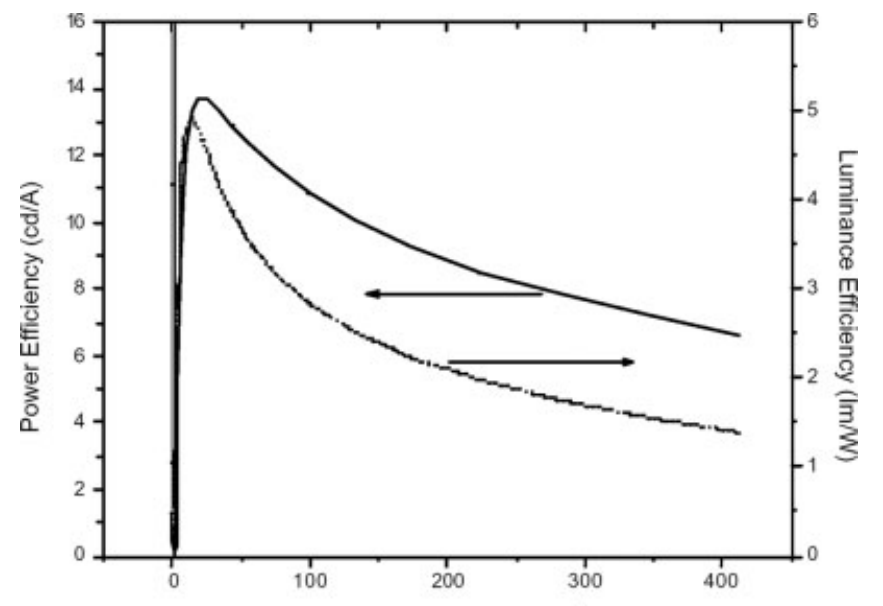

Current Density $\left(\mathrm{mA} / \mathrm{cm}^{2}\right)$

Fig. 4. Power and luminance efficiencies as function of current density of the $\operatorname{Ir}(\text { piq-F })_{2}($ acac $)$ device.

negligible effect from either the $\mathrm{T}-\mathrm{T}$ annihilation or saturation of the excited state. Such an outstanding performance is related to the iridium isoquinoline framework because iridium complexes bearing a 3-(phenyl)isoquinoline ligand show an efficient yellow phosphorescent emission. ${ }^{[20]}$ Characterization of these iridium-isoquinoline complexes to understand the reason behind their remarkable photophysical properties is under investigation.

\section{Experimental}

1-(Phenyl)isoquinoline (piq): To a diethyl ether solution $(12 \mathrm{~mL})$ of isoquinoline was added phenylmagnesium bromide $(2.5 \mathrm{M}$ in $\mathrm{THF}, 2.55 \mathrm{~mL})$ at $23^{\circ} \mathrm{C}$, and the mixture was stirred for $36 \mathrm{~h}$ before treatment with $\mathrm{NH}_{4} \mathrm{Cl}$ solution $(10 \mathrm{~mL})$. The diethyl ether layer was separated, concentrated, and eluted through a silica column to afford 1-(phenyl)isoquinoline as a colorless solid $(0.54 \mathrm{~g}, 2.63 \mathrm{mmol}, 41 \%)$. IR (Nujol) [ $\left.\mathrm{cm}^{-1}\right]: 2960(\mathrm{~m}), 2922(\mathrm{~m}), 2866(\mathrm{~m})$, 2853(m), 1577(s), 1541(s), 1394(s), 1350(s), 1188(s). ${ }^{1} \mathrm{H}$ NMR $\left(\mathrm{CDCl}_{3}\right.$, $400 \mathrm{MHz})[\mathrm{ppm}]: \delta 8.58(\mathrm{~d}, J=6 \mathrm{~Hz} 1 \mathrm{H}) ; 8.09(\mathrm{~d}, J=8 \mathrm{~Hz} 1 \mathrm{H}) ; 7.86(\mathrm{~d}, J=8 \mathrm{~Hz}$ $1 \mathrm{H}) ; 7.68(\mathrm{~d}, J=6.8 \mathrm{~Hz} 1 \mathrm{H}) ; 7.63(\mathrm{t}, J=5.2 \mathrm{~Hz} 1 \mathrm{H}) ; 7.51(\mathrm{~m}, 1 \mathrm{H}) .{ }^{13} \mathrm{C}$ NMR $\left(\mathrm{CDCl}_{3}, 400 \mathrm{MHz}\right)[\mathrm{ppm}]: \delta 160.4,142.2,136.8,129.9,129.9,128.53,128.3$, 127.5, 127.1, 126.9, 126.6, 119.8 .

1-(4'-Fluorophenyl)isoquinoline (piq-F): This compound was prepared similarly to the procedure described for the piq ligand from isoquinoline and 4 fluorophenylmegnesium bromide, and 2-(4'-fluorophenyl)isoquinoline was obtained as a colorless solid in $50 \%$ yield. IR (Nujol) $\left[\mathrm{cm}^{-1}\right]: 2962(\mathrm{~m}), 2921(\mathrm{~m})$, 2865(m), 2852(m), 1576(s), 1540(s), 1393(s), 1351(s), 1188(s). ${ }^{1} \mathrm{H}$ NMR $\left(\mathrm{CDCl}_{3}\right.$ $400 \mathrm{MHz})[\mathrm{ppm}]: \delta 8.58(\mathrm{~d}, J=6.0 \mathrm{~Hz}, 1 \mathrm{H}), 8.06(\mathrm{~d}, J=8.8 \mathrm{~Hz}, 1 \mathrm{H}), 7.89(\mathrm{~d}$, $J=8.0 \mathrm{~Hz}, 1 \mathrm{H}), 7.64-7.72(\mathrm{~m}, 4 \mathrm{H}), 7.55(\mathrm{t}, J=7.2 \mathrm{~Hz}, 1 \mathrm{H}), 7.22(\mathrm{t}, J=8 \mathrm{~Hz}, 2 \mathrm{H})$ ${ }^{13} \mathrm{C} \mathrm{NMR}\left(\mathrm{CDCl}_{3}, 400 \mathrm{MHz}\right)[\mathrm{ppm}]: \delta 162.9\left(\mathrm{~d}, J_{C-F}=246.6 \mathrm{~Hz}\right), 159.4,141.9$, $131.6\left(\mathrm{~d}, J_{C-F}=8.4 \mathrm{~Hz}\right), 129.9,127.0,126.9,126.4,119.9,115.1\left(\mathrm{~d}, J_{C-}\right.$ $\left.F_{F}=21.4 \mathrm{~Hz}\right)$. High-resolution mass spectrometry (HRMS) $(70 \mathrm{eV}$, electron impact (EI)): Calcd. for $\mathrm{C}_{15} \mathrm{H}_{10} \mathrm{NF}$ : 223.0797. Found $\mathrm{C}_{15} \mathrm{H}_{10} \mathrm{NF}: 223.0795$.

5-(4'-Fluorophenyl)-(3,4-benzoquinoline) ( $p b q-F)$ : This compound was prepared similarly to the procedure described for the piq ligand above from 3,4benzoquinoline $(3.00 \mathrm{~g}, 16.7 \mathrm{mmol})$ and 4-fluorophenylmagnesium bromide $(25.1 \mathrm{mmol})$, and colorless solid was obtained in $87 \%$ yield after purification from a silica column. To a $\mathrm{CHCl}_{3}$ solution $(50 \mathrm{~mL})$ of this solid $(4.00 \mathrm{~g}$, $14.6 \mathrm{mmol})$ was added $\mathrm{MnO}_{2}(40 \mathrm{~g})$ at $23^{\circ} \mathrm{C}$, and the mixtures were stirred for $24 \mathrm{~h}$. The solution was filtered, and the filtrate was concentrated, then purified on a silica column to afford 5-(4'-fluorophenyl)-(3,4-benzoquinoline) as a colorless solid (3.70 g, $13.5 \mathrm{mmol})$. IR (Nujol) [ $\left.\mathrm{cm}^{-1}\right]: 2960(\mathrm{~m}), 2920(\mathrm{~m}), 2864(\mathrm{~m})$, $2853(\mathrm{~m}), 1577(\mathrm{~s}), 1541(\mathrm{~s}), 1394(\mathrm{~s}), 1350(\mathrm{~s}), 1188(\mathrm{~s}) .{ }^{1} \mathrm{H} \quad \mathrm{NMR} \quad\left(\mathrm{CDCl}_{3}\right.$, $400 \mathrm{MHz})[\mathrm{ppm}]: \delta 8.69(\mathrm{~d}, J=8.0 \mathrm{~Hz}, 1 \mathrm{H}), 8.60(\mathrm{~d}, J=8.0 \mathrm{~Hz}, 1 \mathrm{H}), 8.22(\mathrm{~d}$, $J=8.0 \mathrm{~Hz}, 1 \mathrm{H}), 8.06(\mathrm{~d}, J=8.4 \mathrm{~Hz}, 1 \mathrm{H}), 7.85(\mathrm{t}, J=7.2 \mathrm{~Hz}, 1 \mathrm{H}), 7.77-7.66(\mathrm{~m}$, $4 \mathrm{H}), 7.61(\mathrm{t}, J=8.0 \mathrm{~Hz}, 1 \mathrm{H}), 7.25(\mathrm{~d}, J=8.6 \mathrm{~Hz}, 2 \mathrm{H}) .{ }^{13} \mathrm{C} \mathrm{NMR}\left(\mathrm{CDCl}_{3}\right.$,
$100 \mathrm{MHz})[\mathrm{ppm}]: \delta 162.9\left(\mathrm{~d}, J_{\mathrm{C}-\mathrm{F}}=245.8 \mathrm{~Hz}\right), 159.7,143.4,135.6,133.1,131.5$, $131.4,130.3,130.0,128.6,128.2,126.9,126.8,124.7,123.4,122.0,121.7,115.3$, 115.1; HRMS (70 eV, EI): Calcd for $\mathrm{C}_{19} \mathrm{H}_{12} \mathrm{FN}$ : 273.3045 . Found: 273.3043

$\operatorname{Ir}(\text { piq })_{2}$ (acac): To a mixed solvent of ethoxyethanol $(9.0 \mathrm{~mL})$ and water $(3 \mathrm{~mL})$ was added $\mathrm{IrCl}_{3} \cdot n \mathrm{H}_{2} \mathrm{O}(0.69 \mathrm{~g}, 84.7$ wt.-\% $\mathrm{Ir}$ content $)$ and piq (1.00 g, $4.88 \mathrm{mmol}$ ), and the mixture was heated at $130^{\circ} \mathrm{C}$ for $24 \mathrm{~h}$. The resulting orange solution was concentrated at $60^{\circ} \mathrm{C}$ under vacuum and the remaining orange solid was collected by filtration, washed with water, hexane, and finally with diethyl ether. The weight of this solid was $1.15 \mathrm{~g}(0.90 \mathrm{mmol}, 92 \%)$. To an ethoxyethanol $(12 \mathrm{~mL})$ of this solid $(1.0 \mathrm{~g}, 0.79 \mathrm{mmol})$ was added acetylacetone $(0.58 \mathrm{~mL})$ and $\mathrm{Na}_{2} \mathrm{CO}_{3}(410 \mathrm{mg})$, and the mixture was heated at $100{ }^{\circ} \mathrm{C}$ for $24 \mathrm{~h}$. The resulting red solution was concentrated at $60^{\circ} \mathrm{C}$ under vacuum and the residues were eluted through a silica column to afford $\operatorname{Ir}(\mathrm{piq})_{2}(\mathrm{acac})$ as a red solid $(0.30 \mathrm{~g}, 0.42 \mathrm{mmol}, 28 \%)$. IR (Nujol) [ $\left.\mathrm{cm}^{-1}\right]: 2964(\mathrm{~m}), 2920(\mathrm{~m})$, 2866(m), 2851(m), 1574(s), 1542(s), 1390(s), 1351(s), 1188(s). ${ }^{1} \mathrm{H}$ NMR (CDCl 3 , $400 \mathrm{MHz})[\mathrm{ppm}]: \delta 8.97(\mathrm{~d}, J=9.2 \mathrm{~Hz}, 2 \mathrm{H}) ; 8.44(\mathrm{~d}, J=6.8 \mathrm{~Hz}, 2 \mathrm{H}) ; 8.18(\mathrm{~d}$, $J=8.0 \mathrm{~Hz} 2 \mathrm{H}) ; 7.91(\mathrm{~m}, 1 \mathrm{H}) ; 7.69(\mathrm{~m}, 1 \mathrm{H}) ; 7.45(\mathrm{~d}, J=6.4 \mathrm{~Hz} \mathrm{1H}) ; 6.87(\mathrm{t}$, $J=6.4 \mathrm{~Hz} 1 \mathrm{H}) ; 6.37(\mathrm{~d}, J=7.2 \mathrm{~Hz} 1 \mathrm{H}) ; 5.18(\mathrm{~s}, 1 \mathrm{H}) ; 1.74(\mathrm{~s}, 6 \mathrm{H}) .{ }^{13} \mathrm{C} \mathrm{NMR}$ $\left(\mathrm{CD}_{2} \mathrm{Cl}_{2}, 400 \mathrm{MHz}\right)[\mathrm{ppm}]: \delta 185.30,169.13,151.98,147.23,140.82,137.64$, $133.98,121.17,130.10,129.12,128.22,127.69,126.97,126.71,120.85,150.60$, 100.89, 28.68. Anal. calcd. for $\mathrm{C}_{35} \mathrm{H}_{27} \mathrm{IrN}_{2} \mathrm{O}_{2}$ : C, 60.07; H, 3.89; N, 4.00. Found: C, 59.89; H, 3.78; N, 3.89 .

$\operatorname{Ir}(\text { piq- } F)_{2}($ acac $)$ : This compound was prepared from 1-(4'-fluorophenyl)isoquinoline (piq-F) and $\mathrm{IrCl}_{3} \cdot n \mathrm{H}_{2} \mathrm{O}$ according to the procedure described for $\operatorname{Ir}(\mathrm{piq})_{2}$ (acac). IR (Nujol) $\left[\mathrm{cm}^{-1}\right]: 2952(\mathrm{~m}), 2926(\mathrm{~m}), 2853(\mathrm{~m}), 2853(\mathrm{~m}), 1575(\mathrm{~s})$, 1507(s), 1456(s), 1270(s). ${ }^{1} \mathrm{H}$ NMR $\left(\mathrm{CD}_{2} \mathrm{Cl}_{2}, 400 \mathrm{MHz}\right)[\mathrm{ppm}]: \delta 8.90(\mathrm{dd}$, $J=4.8,2.4 \mathrm{~Hz}, 2 \mathrm{H}) ; 8.35(\mathrm{~d}, J=5.2 \mathrm{~Hz}, 2 \mathrm{H}), 8.24(\mathrm{dd}, J=7.2,4.2 \mathrm{~Hz}, 2 \mathrm{H}), 7.98$ $(\mathrm{dd}, J=4.4,3.0 \mathrm{~Hz} 2 \mathrm{H}), 7.74-7.77(\mathrm{~m}, 4 \mathrm{H}), 7.55(\mathrm{~d}, J=5.2 \mathrm{~Hz}, 2 \mathrm{H}), 6.66(\mathrm{td}$, $J=8.0,2.0 \mathrm{~Hz}, 2 \mathrm{H}), 5.96(\mathrm{dd}, J=7.6,2.0,2 \mathrm{H}), 5.27(\mathrm{~s}, 1 \mathrm{H}), 1.77(\mathrm{~s}, 6 \mathrm{H}) .{ }^{13} \mathrm{C}$ NMR $\left(\mathrm{CD}_{2} \mathrm{Cl}_{2}, 400 \mathrm{MHz}\right)[\mathrm{ppm}]: \delta 185.4,168.0,162.7\left(\mathrm{~d}, J_{\mathrm{C}-\mathrm{F}}=203.1 \mathrm{~Hz}\right)$, $155.2\left(\mathrm{~d}, J_{\mathrm{C}-\mathrm{F}}=4.4 \mathrm{~Hz}\right), 143.37,140.47,137.72,131.66\left(\mathrm{~d}, J_{\mathrm{C}-\mathrm{F}}=7.1 \mathrm{~Hz}\right), 131.38$, 128.46, 127.78, 126.68, 126.42, 120.60, $119.66(\mathrm{~d}, J=13.4 \mathrm{~Hz}), \quad 108.06$ $(\mathrm{d}, J=18.6 \mathrm{~Hz}), 100.96,28.62$. Anal. calcd for $\mathrm{C}_{35} \mathrm{H}_{25} \mathrm{IrF}_{2} \mathrm{~N}_{2} \mathrm{O}_{2}: \mathrm{C}, 57.13 ; \mathrm{H}$, 3.42 ; N. 3.81. Found: C, 57.01; H, 3.33; N, 3.66 .

$\operatorname{Ir}(\mathrm{pbq}-\mathrm{F})_{2}(\mathrm{acac})$ : This compound was prepared from 5-(4'-fluorophenyl) (3,4-benzoquinoline) (pbq-F) and $\mathrm{IrCl}_{3} \cdot n \mathrm{H}_{2} \mathrm{O}$ according to the procedure described for the complex $\operatorname{Ir}(\text { piq-F) })_{2}$ (acac). IR (Nujol) $\left[\mathrm{cm}^{-1}\right]: 2961(\mathrm{~m}), 2940(\mathrm{~m})$, 2848(m), 2850(m), 1572(s), 1514(s), 1453(s), 1266(s). ${ }^{1} \mathrm{H}$ NMR $\left(\mathrm{CDCl}_{3}\right.$, $400 \mathrm{MHz})[\mathrm{ppm}]: \delta 9.01(\mathrm{~d}, J=8.8 \mathrm{~Hz}, 2 \mathrm{H}), 8.67(\mathrm{~d}, J=8.0 \mathrm{~Hz}, 2 \mathrm{H}), 8.49$ $(\mathrm{d}, J=7.6 \mathrm{~Hz}, 2 \mathrm{H}), 8.31(\mathrm{~d}, J=8.0 \mathrm{~Hz}, 2 \mathrm{H}), 8.19(\mathrm{dd}, J=5.6 \mathrm{~Hz}, J=8.8 \mathrm{~Hz}, 2 \mathrm{H})$, $7.90(\mathrm{t}, J=7.6 \mathrm{~Hz}, 2 \mathrm{H}), 7.78(\mathrm{t}, J=8.4 \mathrm{~Hz}, 2 \mathrm{H}), 7.56(\mathrm{~d}, J=7.2 \mathrm{~Hz}, 2 \mathrm{H}), 7.44$ $(\mathrm{t}, J=8.0 \mathrm{~Hz}, 2 \mathrm{H}), 6.73(\mathrm{dt}, J=3.2 \mathrm{~Hz}, J=8.8 \mathrm{~Hz}, 2 \mathrm{H}), 6.31(\mathrm{dd}, J=3.2 \mathrm{~Hz}$, $J=9.6 \mathrm{~Hz}, 2 \mathrm{H}), 4.43(\mathrm{~s}, 1 \mathrm{H}), 1.28(\mathrm{~s}, 6 \mathrm{H}) .{ }^{13} \mathrm{C} \mathrm{NMR}\left(\mathrm{CDCl}_{3}, 100 \mathrm{MHz}\right)[\mathrm{ppm}]:$ $\delta 185.3,172.0,161.8\left(\mathrm{~d}, J_{\mathrm{C}-\mathrm{F}}=245.8 \mathrm{~Hz}\right), 155.4,145.0,143.9,133.7,133.1,133.0$, 131.5, 129.1, 128.9, 127.1, 126.3, 124.7, 123.3, 123.1, 122.6, 122.4, 121.8, 99.9, 27.7 Anal. calcd for $\mathrm{C}_{43} \mathrm{H}_{29} \mathrm{~F}_{2} \mathrm{IrN}_{2} \mathrm{O}_{2}$ : C, 61.78; H, 3.50; N, 3.35. Found: C, 61.77; H, $3.44 ; \mathrm{N}, 3.40$.

$\operatorname{Ir}(\text { piq- } F)_{3}$ : To a Pyrex tube charged with diiridium complex (300 mg, $0.223 \mathrm{mmol}$ ), piq-F (498 mg, $2.23 \mathrm{mmol})$ and $\mathrm{AgCF}_{3} \mathrm{SO}_{3}(115 \mathrm{mg}, 0.48 \mathrm{mmol}$ ) were added, and the mixture heated at $200{ }^{\circ} \mathrm{C}$ for $24 \mathrm{~h}$. The resulting solid was purified on a silica column to afford $\operatorname{Ir}(\text { piq-F })_{3}$ as a red solid $(40 \mathrm{mg}$, $0.047 \mathrm{mmol}, 21 \%)$. IR (Nujol) $\left[\mathrm{cm}^{-1}\right]: 2944(\mathrm{~m}), 2924(\mathrm{~m}), 2860(\mathrm{~m}), 2850(\mathrm{~m})$, 1574(s), 1500(s), 1444(s), 1268(s). ${ }^{1} \mathrm{H}$ NMR $\left(\mathrm{CDCl}_{3}, 400 \mathrm{MHz}\right)$ [ppm]: 8.85$8.86 \mathrm{~m}, 3 \mathrm{H}) ; 8.15(\mathrm{dd}, J=7.2,4.4 \mathrm{~Hz}, 3 \mathrm{H}), 7.70-7.73(\mathrm{~m}, 3 \mathrm{H}), 7.63-7.65(\mathrm{~m}, 6 \mathrm{H})$, $7.27(\mathrm{~d}, J=5.2 \mathrm{~Hz} 3 \mathrm{H}), 7.11(\mathrm{~d}, J=2.4 \mathrm{~Hz} 3 \mathrm{H}), 6.67(\mathrm{td}, J=7.2,2.4 \mathrm{~Hz}, 3 \mathrm{H})$, $6.58(\mathrm{dd}, J=8.0,2.0 \mathrm{~Hz}, 3 \mathrm{H}) .{ }^{13} \mathrm{C} \mathrm{NMR}\left(\mathrm{CDCl}_{3}, 400 \mathrm{MHz}\right)[\mathrm{ppm}]: \delta 167.2$ $(\mathrm{d}, J=4.5 \mathrm{~Hz}), 166.5,163.9\left(\mathrm{~d}, J_{\mathrm{C}-\mathrm{F}}=203.2 \mathrm{~Hz}\right), 141.3,139.54,136.6,131.7(\mathrm{~d}$, $\left.J_{\mathrm{C}-\mathrm{F}}=7.1 \mathrm{~Hz}\right), 130.4,127.7,127.4,127.1,126.3,122.4\left(\mathrm{~d}, J_{\mathrm{C}-\mathrm{F}}=13.3 \mathrm{~Hz}\right), 120.20$, 107.28(d, $\left.J_{\mathrm{C}-\mathrm{F}}=17.8 \mathrm{~Hz}\right)$. Anal. calcd for $\mathrm{C}_{45} \mathrm{H}_{27} \mathrm{IrF}_{3} \mathrm{~N}_{3}: \mathrm{C}, 62.93 ; \mathrm{H}, 3.17$; N, 4.89. Found: C, 62.88; H, 3.10; N, 4.77.

Measurement of Lifetime and Quantum Yields: Steady-state absorption and emission spectra were recorded by a Hitachi (U-3310) spectrophotometer and an Edinburgh (FS920) fluorimeter, respectively. Both wavelength-dependent excitation and emission response of the fluorimeter have been calibrated. A configuration of front-face excitation was used to measure the emission of the solid sample, in which the cell was made by assembling two edge-polished quartz plates with various Teflon spacers. A combination of appropriate filters was used to avoid the interference from the scattered light.

Lifetime studies were performed by an Edinburgh FL 900 photon-counting system with a hydrogen-filled/or a nitrogen lamp as the excitation source. Data were analyzed using the nonlinear least-squares procedure in combination with an iterative convolution method [21]. Single exponential emission decay was obtained for all studied compounds.

Quinine sulfate/1.0 $\mathrm{N} \mathrm{H}_{2} \mathrm{SO}_{4}$ was used as a reference, assuming a fluorescence quantum yield of 0.564 with $360 \mathrm{~nm}$ excitation [1], to determined quantum yields of the studied compounds in solution. Solution samples were degassed by three freeze-pump-thaw cycles under vigorous stirring. 
OLED Fabrication and Measurements: Pre-patterned indium tin oxide (ITO) substrates with an effective individual device area of $3.14 \mathrm{~mm}^{2}$ were cleaned by sonication in a detergent solution for $3 \mathrm{~min}$ and then washed with large amount of doubly distilled water. Further sonication in ethanol for $3 \mathrm{~min}$ followed before blowing dry with a stream of nitrogen. The ITO substrates were then treated with $\mathrm{O}_{2}$ plasma for one minute before being loaded into the vacuum chamber. The organic layers were deposited thermally at a rate of $0.1-$ $0.3 \mathrm{~nm} \mathrm{~s}^{-1}$ under a pressure of $\sim 2 \times 10^{-5}$ torr in an Ulvac Cryogenic deposition system. An alloy of $\mathrm{Mg}$ and $\mathrm{Ag}$ (ca. 10:1, $50 \mathrm{~nm}$ ) was deposited as the cathode, which was capped with $100 \mathrm{~nm}$ of $\mathrm{Ag}$. The current-voltage-luminance was measured in ambient with a Keithley 2400 Source meter and a Newport $1835 \mathrm{C}$ Optical meter equipped with $818 \mathrm{ST}$ silicon photodiode

Received: November 18, 2002 Final version: February 20, 2003

[1] C. W. Tang, S. A. VanSlyke, Appl. Phys. Lett. 1987, 51, 913.

[2] C. W. Tang, S. A. VanSlyke, C. H. Chen, J. Appl. Phys. 1989, 65, 3610.

[3] V. Bulovic, P. E. Burrows, S. R. Forrest, Semicond. Semimet. 2000, 64, 255.

[4] M. A. Baldo, D. F. O'Brien, Y. You, A. Shoustikov, S. Sibley, M. E. Thompson, S. R. Forrest, Nature 1998, 395, 151.

[5] a) J. L. Fox, C. H. Chen, US Patent 4736032 , 1998. b) T. Inoe, K. Nakatani, JP Patent 6009952,1994 . c) J. Ito, JP Patent 7166160, 1995.

[6] C. W. Tang, SID Symp. Dig. 1996, 27, 181.

[7] Y. Hamada, H. Kanno, T. Tsujioka, H. Takahashi, T. Usuki, Appl. Phys. Lett. 1999, 75, 1682.

[8] P. E. Burrows, S. R. Forrest, S. P. Sibley, M. E. Thompson, Appl. Phys. Lett. 1996, 69, 2959.

[9] Y. Hamada, IEEE Trans. Electron Devices 1997, 44, 1208.

[10] Y. Sakakibara, S. Okutsu, T. Enokida, T. Tani, Appl. Phys. Lett. 1999, 74, 2587.

[11] For recent papers on electrophosphorescence from iridium complexes, see a) B. W. D'Andrade, J. Brooks, V. Adamovich, M. E. Thompson, S. R. Forrest, Adv. Mater. 2002, 14, 1032. b) W. D'Andrade, M. E. Thompson, S. R. Forrest, Adv. Mater. 2002, 14, 147. c) F. C. Chen, Y. Yang, Appl. Phys. Lett. 2002, 80, 2308. d) J. Brooks, Y. Babayan, S. Lamansky, P. I. Djurovich, I. Tsyba, R. Bau, M. E. Thompson, Inorg. Chem. 2002, 41, 3055. e) D. Kolosov, V. Adamovich, P. Djurovich, M. E. Thompson, C. Adachi, J. Am. Chem. Soc. 2002, 124, 9945. f) C. Adachi, M. A. Baldo, M. E. Thompson, S. R. Forrest, J. Appl. Phys. 2001, 90, 5048. g) V. Adamovich, J. Brooks, A. Tamayo, A. M. Alexander, P. I. Djurovich, B. W. D'Andrade, C. Adachi, S. R. Forrest, M. E. Thompson, New J. Chem. 2002, 1171

[12] a) J. Kido, H. Hayase, K. Kongawa, K. Hagai, K. Okamoto, Appl. Phys. Lett. 1994, 65, 2124. b) C. Adachi, M. A. Baldo, S. R. Forrest, J. Appl. Phys. 2000, 87, 8049 .

[13] M. A. Baldo, M. E. Thompson, S. R. Forrest, Nature 2000, 403, 750.

[14] C. Adachi, M. A. Baldo, S. R. Forrest, S. Lamansky, M. E. Thompson, R. C. Kwong, Appl. Phys. Lett. 2001, 78, 1622.

[15] V. V. Grushin, N. Nerron, D. D. Leclous, W. J. Marshall, V. A. Petrov, Y. Wang, Chem. Commun. 2001, 1494.

16] S. Lamansky, P. Djurovich, D. Murphy, F. Abdel-Razzaq, H.-E. Lee, C. Adachi, P. E. Burrows, S. R. Forrest, M. E. Thompson, J. Am. Chem. Soc. 2001, 123, 4304.

17] M. G. Colombo, A. Hauser, H. U. Gudel, Inorg. Chem 1993, 32, 3088.

[18] B. Schimid, F. O. Garces, R. J. Watts, Inorg. Chem. 1994, 33, 9.

[19] The related $\operatorname{Ir}(\mathrm{piq})_{3}$ complex was briefly mentioned in a recent conference paper by S. Ohada and co-workers. The device based on this complex gave a maximum bightness $10000 \mathrm{~cd} \mathrm{~m}^{-2}$ at $J=365 \mathrm{~mA} \mathrm{~cm}^{-2}$. See: S. Okada, H. Iwawak, M. Furugori, J. Kamatani, S. Lgawa, T. Moriyama, S. Miuva, A. Tsuboyama, T. Takiguchi, H. Mizutani, SID Symp. Dig. 2002, 1360.

[20] The red iridium phosphors in this study have a 1-(phenyl)isoquinoline framework. In our new findings, iridium complexes bearing a 3-(phenyl)isoquinoline framework also show an efficient yellow phosphorescence emission $(565 \mathrm{~nm})$ with a maximum brightness $65000 \mathrm{~cd} \mathrm{~m}^{-2}$. C.-L. Li, R.-S. Liu, unpublished.

[21] J. N. Demas, G. A. Crosby, J. Phys. Chem. 1971, 75, 991.

\section{Cell-Responsive Synthetic Hydrogels**}

\author{
By Matthias P. Lutolf, George P. Raeber, Andreas H. Zisch, \\ Nicola Tirelli, and Jeffrey A. Hubbell*
}

An important part of tissue function relies on the dynamic dialog that exists between cells and their extracellular matrices (ECM): the ECM is active, presenting bound adhesion sites that interact with cell-surface receptors, ${ }^{[1]}$ and it is responsive to signals presented by cells, locally degrading under the influence of proteases at the surface of the migrating cell. ${ }^{[2-4]}$ While artificial biomaterial matrices used in cell culture and tissue engineering have been developed to be responsive to physical ${ }^{[5-9]}$ and even biochemical ${ }^{[10-14]}$ stimuli, they do not widely address this concept of responsiveness to cellular stimuli. We mimicked these features in synthetic hydrogel networks, for application in both cell biology and tissue engineering, ${ }^{[15-17]}$ incorporating pendant receptor-binding sites so that cells can exert traction ${ }^{[18]}$ and crosslinking protease-sensitive degradation sites so that cells can create a path to enable forward movement. ${ }^{[19]}$ Our results demonstrate that synthetic materials can be responsive to cellular influences in vitro and in vivo, including here, as an example, inducing and responding to angiogenic invasion.

We formed polymer networks in situ using conjugate addition reactions. We linked molecular building blocks performing either structural (poly(ethylene glycol), PEG) or biological (oligopeptides) function (Scheme 1). Vinyl sulfone (VS)-functionalized multiarmed telechelic PEG macromers reacted with thiolate groups of cysteine-containing peptides by conjugate addition, ${ }^{[20]}$ a reaction that is highly selective versus biological amines ${ }^{[21]}$ and can be carried out at physiological temperature and near physiological $\mathrm{pH}$.

We selected PEG-based macromers as the main structural component due to their hydrophilicity and resistance to protein adsorption. ${ }^{[22,23]}$ We chose bis-cysteine peptides as one biological component, since upon reaction they become part of a crosslinked network structure and contribute to its mechanical character, i.e., elastically active. As a result, any designed sensitivity of these building blocks to cellular proteases

[*] Prof. J. A. Hubbell, M. P. Lutolf, G. P. Raeber, Dr. A. H. Zisch, Dr. N. Tirelli Institute for Biomedical Engineering and Department of Materials Science Swiss Federal Institute of Technology Zurich (ETHZ) and University of Zurich

Moussonstrasse 18, CH-8044 Zurich (Switzerland) E-mail: hubbell@biomed.mat.ethz.ch

[**] This work was funded by a grant from the Swiss National Science Foundation and the European Union Fifth Framework. MMP-1 was a generous gift from Dr. G. B. Fields, Florida Atlantic University, Boca Raton, FL (USA) and Dr. H. Nagase, Imperial College of Science, Technology and Medicine, London (UK). Polyurethane sponges were a generous gift from Dr. D. Bezuidenhout and Dr. Zilla, Cape Heart Center, University of Cape Town, South Africa. We thank Dr. M. Höchli and Dr. T. Bächi of the Electron Microscopy Laboratory (EMZ) at the University of Zurich for help with CLSM. 